\title{
Achieving 100 percent compliance to perioperative antibiotic administration: a quality improvement initiative
}

\author{
Sui An $\underline{\mathrm{Lie}}^{1}$, MBBS, MMed, Kwang Yang Aaron $\underline{\mathrm{Lee}}^{1}$, MBBS, MMed, Meng Huat $\underline{G o h}^{1}$, MBBS, FAnzCA, Sudha Harikrishnan ${ }^{1}$,
} Ruban Poopalalingam ${ }^{1}$, MBBS, MMed

INTRODUCTION Timely administration of prophylactic antibiotics within 60 minutes before surgical incision is important for reducing surgical site infections. This quality improvement initiative aimed to work towards achieving $100 \%$ compliance with perioperative antibiotic administration.

METHODS We examined the workflow in our Anaesthesia Information Management System (AIMS) and proposed interventions using cause-and-effect analysis of anonymised anaesthetic records from eligible surgical cases extracted from AIMS. This ultimately led to the implementation of an antibiotic pop-up reminder. The overall process was done in a few small plan-do-study-act cycles involving raising awareness, education and reorganisation of AIMS before implementation of the antibiotic pop-up reminder. Data analysis took place from August 2014 to September 2016. Compliance was defined as documented antibiotic administration within 60 minutes before surgical incision, or as documented reason for omission. RESULTS The median monthly compliance rate, for 33,038 cases before and 28,315 cases after the reminder was implemented, increased from $67.0 \%$ at baseline to $94.5 \%$. This increase was consistent and sustained for a year despite frequent personnel turnover. Documentation of antibiotic administration also improved from $81.7 \%$ to $99.3 \%$, allowing us to identify and address novel problems that were initially not apparent, and resulting in several department recommendations. These included administering antibiotics later for cases with predicted longer-than-expected preparation times and bringing forward antibiotic administration in lower-segment Caesarean sections.

CONCLUSION The use of information technology and implementation of an antibiotic pop-up reminder on AIMS streamlined our work processes and brought us closer to achieving $100 \%$ on-time compliance with perioperative antibiotic administration.

Keywords: antibiotic prophylaxis, documentation, guideline adherence, reminder system, surgical wound infection

\section{INTRODUCTION}

Timely perioperative antibiotic administration is viewed as an important tenet for reducing surgical site infections (SSIs) and is included in many international evidence-based guidelines. ${ }^{(1-3)}$ These guidelines recommend that prophylactic antibiotics should be administered within the 30-60 minutes prior to surgical incision. The rationale is to allow time to establish adequate tissue and serum antibiotic levels by the time of skin incision. Prevention of SSIs is the focus and priority of the Surgical Care Improvement Project (SCIP). A set of SCIP initiatives, published jointly by the United States' Centers for Medicare and Medicaid Services and the Joint Commission, ${ }^{(4,5)}$ aims to standardise documentation and track compliance with various SCIP initiatives.

At our institution, anaesthesiologists are major process owners involved in perioperative antibiotic administration. They administer prophylactic antibiotics in the operating theatre at the time of anaesthesia induction. The choice of antibiotics is based on the hospital's published antibiotic guidelines. The difficulties we faced in adhering to the SCIP initiatives were twofold: firstly, antibiotic administration is often superseded by other aspects during anaesthesia induction; secondly, it often becomes difficult to keep track of and remember the redosing timings as the case progresses. A preliminary analysis of our Anaesthesia Information Management System (AIMS) records of all eligible surgical cases from August 2014 to November 2014 showed that our median baseline compliance to timely perioperative antibiotic administration was only $67.0 \%$. We found similar reports of low adherence to perioperative antibiotic administration at other centres worldwide, ${ }^{(6-8)}$ highlighting a need for quality improvement.

Learning from the experience of other authors who utilised AIMS, ${ }^{(9-11)}$ we hypothesised that modifying our AIMS platform and workflow would have an impact on perioperative antibiotic compliance. Hence, we embarked on a quality improvement initiative using plan-do-study-act (PDSA) cycles and implementing an antibiotic pop-up reminder on AIMS, aiming to achieve $100 \%$ compliance to administering antibiotic prophylaxis within 0-60 minutes before skin incision over a period of 22 months. The timing of 0-60 minutes before skin incision was adopted based on existing guidelines ${ }^{(1,4,5)}$ and due to pragmatic reasons, such as ease of administration and feasibility in a setting where a high turnover of surgical cases is the norm.

\section{METHODS}

Anonymised anaesthesia records of all eligible surgical cases from August 2014 to October 2014 at Singapore General Hospital, Singapore, were extracted from AIMS for cause-and-effect analysis to identify potential causes of the problem. Data extracted for each

${ }^{1}$ Department of Anaesthesiology, Singapore General Hospital, Singapore

Correspondence: Dr Sui An Lie, Associate Consultant, Department of Anaesthesiology, Singapore General Hospital, Outram Road, Singapore 169608. Lie.sui.an@singhealth.com.sg 


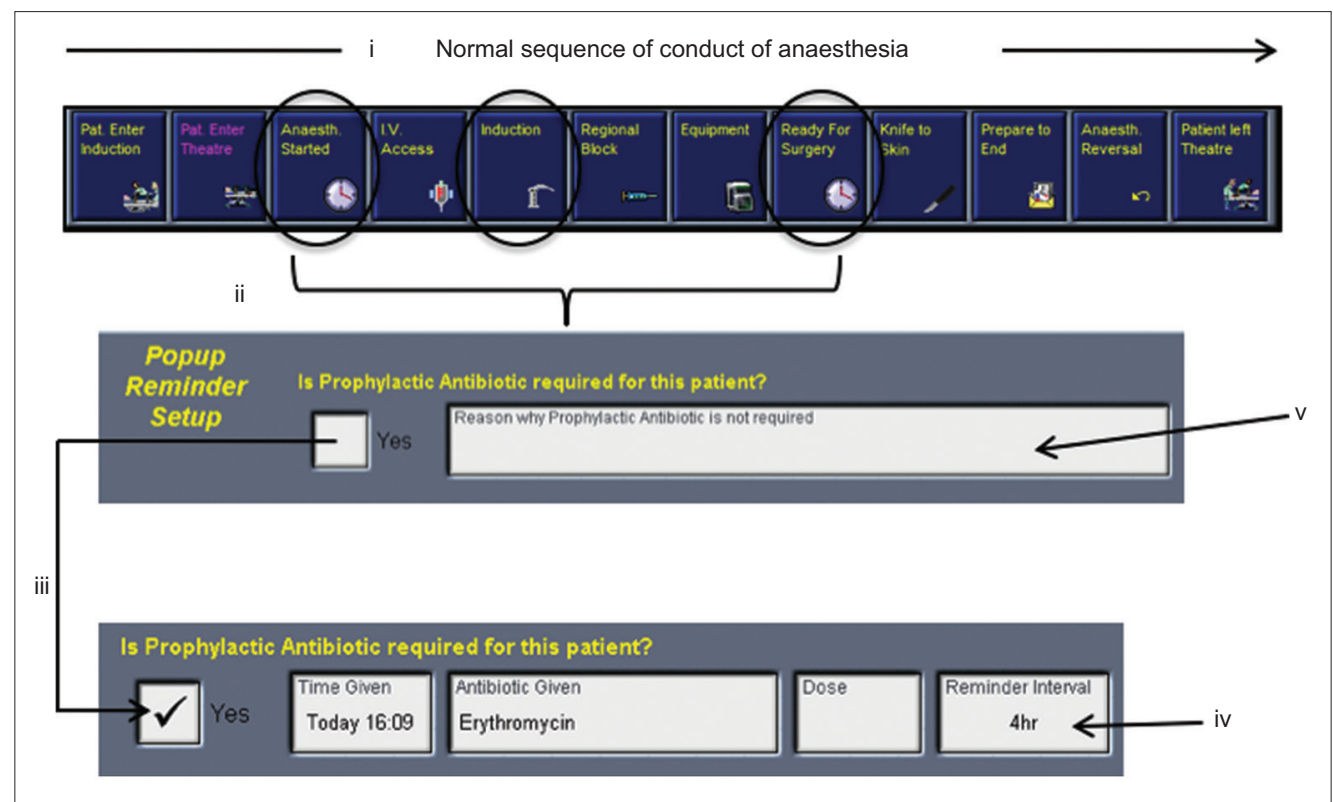

Fig. 1 Intervention workflow for the antibiotic pop-up reminder on the Anaesthesia Information Management System. Diagram shows: (i) event icons arranged in the normal sequence of activities in the course of anaesthesia, i.e. patient enters induction room, patient enters operating theatre (OT), anaesthesia started/intravenous access, induction, ready for surgery, knife to skin, prepare to end, anaesthesia reversal and patient leaves OT; (ii) the antibiotic pop-up reminder being activated when the user clicks the 'ready for surgery', 'anaesthesia started' or 'induction' buttons; and (iii) additional fields being triggered for 'time given', 'antibiotic given', 'dose' and 'reminder interval' if the user selects the option that the patient requires a prophylactic antibiotic. If the system determines that an antibiotic has already been recorded in the medication panel, these fields are auto-populated. (iv) Diagram shows a 'reminder interval' that can be set to prompt the user later when the antibiotic is due for redosing; ( $v$ ) if a prophylactic antibiotic is not required or given, the user needs to select the reason from a drop-down menu. The reason field is mandatory.

month included: location of surgery; procedure type; choice of antibiotic; timing of first dose antibiotic administration relative to surgical procedure start time (or 'knife to skin'); timing of redoses, where applicable; reason for omission, where applicable; practitioner identity; and, subsequently, key performance indicators met or not met. Cases with special administration requirements for prophylactic antibiotics (e.g. vancomycin or fluoroquinolone) were excluded, as these antibiotics require a longer infusion time and had to be started before the patient's arrival in the operating theatre to finish the infusions within 60 minutes of the surgical incision.

From our cause-and-effect analysis, some causes that were identified included lack of knowledge regarding timing of the antibiotic prophylaxis, difficulty in obtaining certain antibiotics, difficulty in anticipating the incision time, uncertainty regarding the choice of antibiotics, and lack of documentation when antibiotic prophylaxis was not required. We determined that the largest contributors were failure to document and lack of a time trigger. Because both events could be linked to AIMS in our workflow, we considered whether modifications to AIMS could improve compliance with antibiotic administration.

We examined the existing AIMS workflow and proposed interventions that would provide reminders and improve compliance without affecting complexity or introducing unnecessary interruptions. The workflow had to be intuitive because the high turnover of our junior staff at irregular intervals meant that extensive training would not be pragmatic. The end product was an antibiotic pop-up reminder with mandatory input from the anaesthesiologist. The reminder was first displayed when the start of anaesthesia was documented on AIMS. The anaesthesiologist could then enter the timing and type of antibiotic administered. If an antibiotic was not required, it was mandatory to provide a reason. The system would later prompt the anaesthesiologist when the antibiotic was due for redosing. In cases where prophylactic antibiotics were not required or given, another prompt asked users to enter a reason at the end of the case. The intervention workflow is summarised in Fig. 1.

After obtaining approval from the department head, we submitted the change request to the AIMS technical support team, and the institution approved the funding for the request. The overall change process involved a few short PDSA cycles that can be largely summarised into three phases. Phase 1 was meant to: (a) raise awareness of current prophylactic antibiotic guidelines available on the intranet; (b) educate staff on the importance of timely antibiotic administration and the need for improved compliance with the AIMS documentation through department meetings and emails; and (c) seek buy-in from the department. Phase 2 aimed to improve the organisation of the AIMS section regarding reasons for not giving antibiotics, to achieve more accurate documentation of reasons (e.g. 'already given in the ward, not due for redosing', 'surgery does not need antibiotics') and expected or unexpected delays (e.g. 'awaiting intraoperative cultures to be taken', 'antibiotic had to be fetched from the ward'). Phases 1 and 2 were implemented from December 2014 to September 2015. Phase 3 involved actual 'live' implementation of the antibiotics pop-up reminder at the end of September 2015 across all operating theatres utilising AIMS.

The field 'surgery start time' or 'knife to skin' was made mandatory and had to be recorded before the 'prepare to end' 
event icon could be pressed, or the document could not be finalised. The event icons on AIMS were rearranged to follow the normal sequence of activities in the course of anaesthesia. Staff were notified and educated about this new pop-up reminder through emails, detailed instructional slides and briefing at department meetings. Individual antibiotic compliance data was sent to department staff to create awareness.

During these phases, data analysis and surveillance were ongoing, and areas that lacked compliance were identified and feedback provided to staff. Data was generated from the AIMS database on a monthly basis. After obtaining the compliance data, we plotted run charts to monitor the compliance rate of prophylactic antibiotic administration over the study period (Fig. 2). A period of four months before the start of any intervention (August 2014-November 2014) was analysed to determine the baseline compliance rate to prophylactic antibiotic administration. Subsequently, the pre-implementation period (comprising Phases 1 and 2) was from December 2014 to September 2015, before the implementation of the antibiotics pop-up reminder in September 2015 (Phase 3). During the post-implementation period, from October 2015 to September 2016, data was analysed to determine if any sustained change in compliance had occurred. Compliance or meeting key performance indicators was defined as documented antibiotic administration within 60 minutes before surgical incision or documentation with reason(s) prophylactic antibiotic was not needed or given. Noncompliance was divided into cases in which dosing was too early, too late or not documented.
Percentage compliance was defined as the number of cases meeting compliance criteria against the total number of cases with anaesthetic care.

\section{RESULTS}

There were a total of 61,353 cases during our study period from August 2014 to September 2016. Out of 33,038 cases at baseline and the pre-implementation period, 6,034 (18.3\%) cases were excluded from analysis due to incomplete data (e.g. surgery start date or time not available). Out of the 28,315 cases in the postimplementation period, 198 (0.7\%) cases were likewise excluded from analysis. There was a sustained increase in the median monthly compliance rate from $67.0 \%$ at baseline to $75.3 \%$ during the pre-implementation period (Phases 1 and 2), and to $94.5 \%$ during the post-implementation period (Fig. 2). Table I shows the average compliance and noncompliance rates of all eligible cases at baseline, and in the pre- and post-implementation periods. There was a large drop in the proportion of 'noncompliant - antibiotic given too late' cases between the baseline and pre-implementation period (from $10.1 \%$ to $2.3 \%$ ). The proportion further decreased to $1.3 \%$ in the post-implementation period. Compared to the pre-implementation period, the proportion of 'noncompliant - antibiotic not given/documented' cases dropped significantly in the post-implementation period (from $20.4 \%$ to $2.0 \%$ ).

Cefazolin was the most commonly used antibiotic (51.5\%) for surgical prophylaxis, followed by ceftriaxone $(10.3 \%)$ and metronidazole $(5.4 \%)$. In cases where antibiotics were

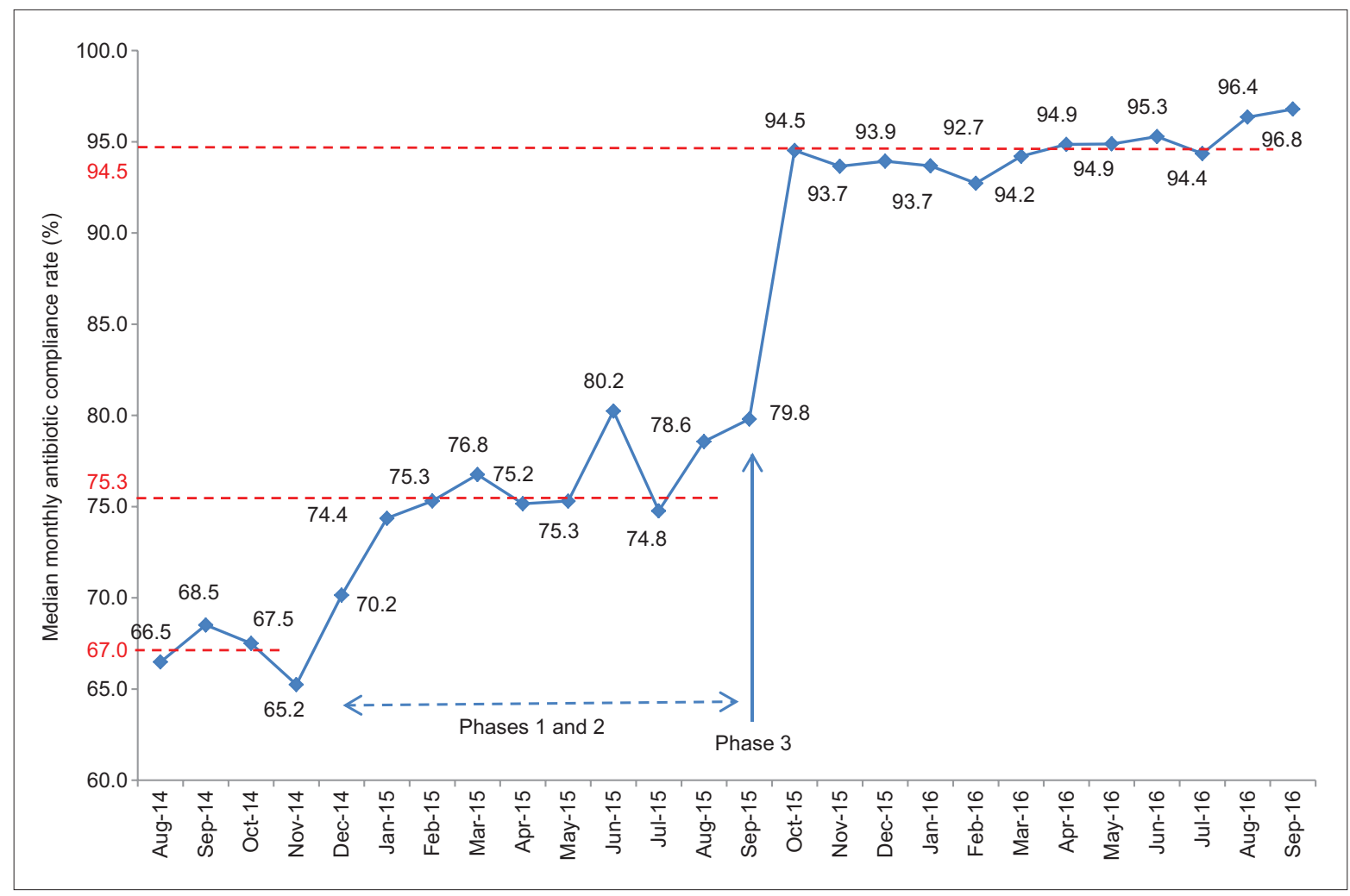

Fig. 2 Run chart shows the median monthly antibiotic compliance rate during the intervention phases, consisting of the baseline period in August-November 2014; Phases 1 and 2 in December 2014-September 2015, before live implementation of the antibiotic pop-up reminder in September 2015; Phase 3 , the actual 'live' implementation of the antibiotic pop-up reminder in September 2015; and the subsequent post-implementation period in October 2015-September 2016. 
Table I. Overall compliance and noncompliance rates to prophylactic antibiotic administration.

\begin{tabular}{|llll|}
\hline Variable & & & \% \\
\cline { 2 - 4 } & Compliant & & Noncompliant \\
\cline { 2 - 4 } & & Given too early & 10.1 \\
\hline Baseline $(n=7,152)$ & 67.0 & 1.5 & 2.3 \\
\hline Pre-implementation period $(n=19,852)$ & 76.1 & 1.2 & 1.3 \\
\hline Post-implementation period $(n=27,919)$ & 95.0 & 1.7 & 20.4 \\
\hline
\end{tabular}

Given too early: antibiotics administered > 60 minutes before surgical incision; given too late: antibiotics administered after surgical incision

Table II. Noncompliance to prophylactic antibiotic administration by surgery type.

\begin{tabular}{|lll|}
\hline \multirow{2}{*}{ Type of surgery } & \multicolumn{2}{c|}{ Noncompliance rate (\%) } \\
\cline { 2 - 3 } & $\begin{array}{l}\text { Pre- } \\
\text { implementation }\end{array}$ & $\begin{array}{l}\text { Post- } \\
\text { implementation }\end{array}$ \\
\hline Obstetrics & 10.4 & 28.7 \\
\hline Gynaecology & 10.3 & 2.3 \\
\hline General* & 13.6 & 11.0 \\
\hline Head and neck & 2.3 & 2.2 \\
\hline $\begin{array}{l}\text { Hepatopancreaticobiliary/ } \\
\text { upper gastrointestinal }\end{array}$ & 4.8 & 3.2 \\
\hline Vascular & 4.3 & \\
\hline Colorectal & 9.6 & 3.7 \\
\hline Neurosurgery & 4.0 & 2.8 \\
\hline Oral and maxillofacial & 1.1 & 9.2 \\
\hline Orthopaedics/hand & 18.0 & 0.9 \\
\hline Otolaryngology/eye & 4.3 & 19.8 \\
\hline Plastic & 5.9 & 3.0 \\
\hline Cardiothoracic & 4.6 & 3.0 \\
\hline Urology & 6.5 & 5.8 \\
\hline Other & & 4.8 \\
\hline
\end{tabular}

* Includes breast surgery and surgical oncology. tIncludes cases done under procedural sedation that do not fit into any surgical categories (e.g. electroconvulsive therapy).

documented as not required, the most often cited reason was that the antibiotic had been given in the ward and was not due for redose.

During the pre-implementation period, orthopaedics and hand surgery $(18.0 \%)$, general surgery including breast surgery and surgical oncology (13.6\%), and obstetrics and gynaecology $(20.7 \%)$ cases constituted the majority of cases of noncompliance to prophylactic antibiotic administration. Contrary to our expectations, the proportion of noncompliant cases in obstetrics and neurosurgery increased markedly after implementation of the antibiotic pop-up reminder, from $10.4 \%$ to $28.7 \%$ and from $4.0 \%$ to $9.2 \%$, respectively (Table II). This was unlike the trend observed across other surgical specialties, suggesting the possible presence of systemic problems outside of documentation of antibiotic administration that were not addressed by the introduction of the pop-up reminder.

Considering this, we classified noncompliant cases based on whether antibiotic administration was not documented, given too late or given too early. Under each category, we further analysed the distribution of noncompliant cases according to surgical specialty (not shown). Among cases that were 'noncompliant antibiotic not documented', a majority (31.3\%) of cases involved emergency surgical cases in the emergency operating room, where data regarding the nature of surgery and antibiotics is often neglected. 'Noncompliant - antibiotic given too late' was most commonly observed in obstetric cases, particularly lower-segment Caesarean sections, which made up 37.9\% of all cases where antibiotic was given late. In our institution, it is a common practice to administer antibiotics only after the baby has been delivered (after the umbilical cord has been clamped), due to concerns that fetal exposure to antibiotics could mask fetal infection and lead to the emergence of resistant bacterial strains. Among cases that were 'noncompliant - antibiotic given too early' (> 60 minutes before surgical incision), most cases (37.3\%) were neurosurgical cases in which a longer-than-expected amount of time post induction was spent positioning and preparing the patient, resulting in antibiotics being administered too early relative to 'knife to skin' timing.

\section{DISCUSSION}

SSI is a common cause of postsurgical morbidity and mortality, and significantly adds to the length of hospitalisation and cost of treatment. ${ }^{(12-14)}$ In addition to measures such as appropriate skin antisepsis and reducing the duration of surgical procedures, international guidelines on SSI prevention also recommend timely administration of prophylactic antibiotics (taken 30-60 minutes before surgical incision), appropriate choice of antibiotics and supplemental dosing of antibiotics for prolonged cases. ${ }^{(1-3)}$ These guidelines also encourage audits to monitor adherence to perioperative antibiotic administration. Compliance rates of perioperative antibiotic administration have now become our department's key performance indicator because of the importance of antibiotic timing and selection for reducing perioperative morbidity and mortality. The SCIP also recommends the reporting of antibiotic timeliness (within one hour before surgical incision) as a quality measure. In this article, we described the implementation of an antibiotic pop-up reminder on AIMS to improve compliance with perioperative prophylactic antibiotic administration.

Previous studies have employed various methods that were incorporated into AIMS to improve antibiotic compliance and our results compare favourably with their findings. Among methods already reported are the use of interactive visual reminders ${ }^{(9)}$ and a computerised reminder system, ${ }^{(15)}$ generating reports from the AIMS database and giving provider-specific 
feedback. ${ }^{(10)}$ Nair et al described a strategy of using "real-time guidance and reminders through electronic messages generated by a computerised decision support system (Smart Anaesthesia Messenger, or SAM) which significantly improved compliance with consistency". (11) Importantly, they showed that the "installation of AIMS itself did not improve antibiotic compliance over that achieved with paper anaesthesia records". ${ }^{(1)}$ Our study mirrored the observations of Nair et al, as the median baseline antibiotic compliance rate was $67.0 \%$ despite the existence of AIMS prior to the intervention.

At the start of our quality improvement initiative, we raised awareness, provided the necessary education and emphasised the importance of solving the problem of poor compliance with antibiotic administration at the department level. This, together with the initial reorganisation of AIMS during Phases 1 and 2, produced a modest increase in median monthly compliance rates, to $75.3 \%$. Expanding on this, we then modified the existing AIMS platform to add an antibiotic pop-up reminder that required input by the anaesthesiologist. This addition reinforced perioperative antibiotic administration, improving the overall compliance rate from $66.5 \%$ in the first month of our study period to $96.8 \%$ in the final month.

The pop-up reminder was effective predominantly because it improved documentation of antibiotic administration: a greater proportion of complete records were available for data analysis in the post-implementation period (99.3\%) compared to the pre-implementation period $(81.7 \%)$, and there was a decrease in the proportion of cases that were not compliant due to lack of documentation (from $20.4 \%$ to $2.0 \%$ ). Consistent and sustainable results were achieved throughout the one-year post-implementation period despite frequent personnel turnover and resident changes. This demonstrates that for a change to be effective and sustainable, it should be re-engineered into a pre-existing workflow, while maintaining ease of use and not compromising on efficiency. The AIMS database also provided us with actionable data for continual improvement after multiple PDSA reviews.

By leveraging the use of electronic documentation and data analytics during the quality improvement initiative, we could identify and address novel problems that were not apparent in the initial planning stage. For example, in cases with predicted longer-than-expected patient preparation times, such as neurosurgical cases or high-risk cases that require setting of invasive lines, anaesthesia providers have been advised to administer prophylactic antibiotics only after all anaesthetic preparation has been completed. Reminders were also given to senior staff covering emergency operating theatres to reinforce the need for strict antibiotic dosing, where applicable, as these cases tended to be higher risk due to their emergent nature. We also approached obstetricians with evidence that strongly advocated the administration of antibiotics before 'knife to skin' (as opposed to the traditional practice of giving antibiotics only after clamping of the umbilical cord) for lower-segment Caesarean sections. ${ }^{(16,17)}$ To further improve compliance through feedback and self-improvement, an individualised quarterly performance report was sent to each anaesthesiologist comparing their personal antibiotic prophylaxis compliance rate with the department average.

A limitation of our quality improvement initiative was that it only focused on the timeliness of antibiotic administration. We anticipate that by adapting AIMS technology, we can also evaluate inconsistencies in the other tenets of perioperative antibiotic administration, namely appropriateness of the choice of antibiotics, redosing times in lengthy surgical procedures and timely administration of prophylactic antibiotics when given as an infusion.

In summary, electronic documentation can provide actionable data for quality improvement in healthcare. Judicious application of information technology can also facilitate work processes and create conducive platforms for change. By adopting multiple PDSA cycles, we were able to implement an antibiotic pop-up reminder on the AIMS database, increasing our compliance with SCIP guidelines on timely administration of prophylactic antibiotics to $96.8 \%$ during the final month of the study period. We learnt that for change to be sustained, it should be incorporated into a pre-existing workflow in a seamless manner, maintaining ease of use and without compromising efficiency.

\section{REFERENCES}

1. Bratzler DW, Houck PM. Antimicrobial prophylaxis for surgery: an advisory statement from the National Surgical Infection Prevention Project. Clin Infect Dis 2004; 38:1706-15.

2. Mangram AJ, Horan TC, Pearson ML, Silver LC, Jarvis WR. Guideline for prevention of surgical site infection, 1999. Centres for Disease Control and Prevention (CDC) Hospital Infection Control Practices Advisory Committee. Am J Infect Control 1999; 27:97-132.

3. Scottish Intercollegiate Guidelines Network. SIGN 104. Antibiotic prophylaxis in surgery. A national clinical guideline. July 2008, updated April 2014. Available at: http://www.sign.ac.uk/assets/sign104.pdf. Accessed July 2, 2017.

4. Specifications Manual, Version 4.3b. Available at: https://www.qualitynet. org/dcs/ContentServer?c=Page\&pagename $=$ QnetPublic $\% 2 \mathrm{FPage} \%$ 2FQnetTier3\&cid=1228773564870. Accessed June 18, 2017.

5. The Joint Commission. Surgical Care Improvement Project Core Measure Set. Available at: http://www.jointcommission.org/assets/1/6/Surgical\%20Care\%20 Improvement\%20Project.pdf. Accessed June 18, 2017.

6. Martin C, Pourriat JL. Quality of perioperative antibiotic administration by French anaesthetists. J Hosp Infect 1998; 40:47-53.

7. Al-Momany NH, Al-Bakri AG, Makahleh ZM, Wazaify MM. Adherence to international antimicrobial prophylaxis guidelines in cardiac surgery: a Jordanian study demonstrates need for quality improvement. J Manag Care Pharm 2009; 15:262-71

8. Young B, Ng TM, Teng C, et al. Nonconcordance with surgical site infection prevention guidelines and rates of surgical site infections for general surgical, neurological, and orthopedic procedures. Antimicrob Agents Chemother 2011; 55:4659-63.

9. Wax DB, Beilin Y, Levin M, et al. The effect of an interactive visual reminder in an anesthesia information management system on timeliness of prophylactic antibiotic administration. Anesth Analg 2007; 104:1462-6.

10. O'Reilly M, Talsma A, VanRiper S, Kheterpal S, Burney R. An anesthesia information system designed to provide physician-specific feedback improves timely administration of prophylactic antibiotics. Anesth Analg 2006; 103:908-12.

11. Nair BG, Newman SF, Peterson GN, Wu WY, Schwid HA. Feedback mechanisms including real-time electronic alerts to achieve near $100 \%$ timely prophylactic antibiotic administration in surgical cases. Anesth Analg 2010; 111:1293-300.

12. de Lissovoy G, Fraeman K, Hutchins V, et al. Surgical site infection: incidence and impact on hospital utilization and treatment costs. Am J Infect Control 2009; 37:387-97.

13. Kirkland KB, Briggs JP, Trivette SL, Wilkinson WE, Sexton DJ. The impact of surgical-site infections in the 1990s: attributable mortality, excess length of hospitalization, and extra costs. Infect Control Hosp Epidemiol 1999; 20:725-30.

14. Vasudevan A, Lee CN, Tambyah PA. Economic impact of surgical site infections 
(SSI) post-coronary artery bypass grafting (CABG) in a tertiary hospital in Singapore [abstract]. Singapore Public Health and Occupational Medicine Conference, 2012.

15. St Jacques $\mathrm{P}$, Sanders $\mathrm{N}$, Patel $\mathrm{N}$, et al. Improving timely surgical antibiotic prophylaxis redosing administration using computerized record prompts. Surg Infect (Larchmt) 2005; 6:215-21.
16. Baaqeel $\mathrm{H}$, Baaqeel R. Timing of administration of prophylactic antibiotics for caesarean section: a systematic review and meta-analysis. BJOG 2013; 120:661-9.

17. Constantine MM, Rahman M, Ghulmiyah L, et al Timing of perioperative antibiotics for cesarean delivery: a metaanalysis. Am J Obstet Gynecol 2008; 199:301.e1-6. 